\section{Configuration and Antiplasmodial Activity}

THE current view ${ }^{1,2}$ that there is no relationship between optical activity (configuration) and antiplasmodial action in the Cinchona series does not appear to be fully justified.

Neither the configuration of the asymmetric centres at $\mathrm{C}_{3}$ and $\mathrm{C}_{4}{ }^{3}$ nor the bridged structure of the quinuclidine skeleton in quinine ${ }^{1}$ has an influence on its activity. This latter statement follows from the fact that niquine and niquidine are biologically active ${ }^{2}$ and that the same is true of the (6-methoxy-quinolyl4)- $\alpha$-piperidyl-carbinol (II) of Ainley and King ${ }^{4}$. The part of the molecule which is crucial for our discussion is, therefore, $\mathrm{C}_{8}$ and $\mathrm{C}_{8}$.

Furthermore, the $l$-alkaloids (with respect to both $\mathrm{C}_{8}$ and $\mathrm{C}_{8}$ ) are, at least frequently, not significantly different in activity from $d$-bases. The quinine equivalents are for :

\begin{tabular}{|c|c|c|c|c|c|}
\hline$d$-apo-quinidine $\mathrm{m}$ & thy & her & $1 \cdot 0$ & l-a-iso-quinine & \\
\hline niquidine $\quad .$. & $\therefore$ & .. & $1 \cdot 5$ & niquine $\quad$. & .. \\
\hline dihydro-niquidine & . & . & $1 \cdot 1$ & dihydro-niquine & \\
\hline dihydro-cupreine & .. & .. & 0.9 & dihydro-cupreidine & \\
\hline dihydro-quinine & .. & . & $1 \cdot 35$ & dihydro-quinidine & .. \\
\hline apo-quinine.. & .. & .. & $1 \cdot 0$ & apo-quinidine & .. \\
\hline
\end{tabular}

Epimerization at $\mathrm{C}_{8}$ alone, however, causes complete loss of activity ${ }^{5}$, whatever the configuration of the active compound epimerized is. One is, therefore, led to the hypothesis that alkaloids with the same sign of partial rotation at $\mathrm{C}_{8}$ and $\mathrm{C}_{9}$ have antiplasmodial activity; those with different sign of rotation at these two centres are inactive ${ }^{2,6}$.

The following two conclusions which can be drawn from these considerations appear to be borne out by experiment.

(a) The quinine equivalents of optical antipodes (with respect to $\mathrm{C}_{8}$ and $\mathrm{C}_{8}$ only) do not differ more than by a factor of $1 \cdot 5-2$. Examples :

Quinine

dihydro-cupreine

epi-C $\mathrm{C}_{\mathrm{s}}$-dihydro-quinine

epi- $\mathrm{C}_{\mathrm{q}}$-quinine

\section{Quinidine}

dihydro-cupreidin

epi-C $\mathrm{C}_{8}$-dihydro-quinidin epi- $\mathrm{C}_{9}$-quinidine, etc.

(b) The quinine equivalents of diastereomerides (with respect to $\mathrm{C}_{8}$ and $\mathrm{C}_{9}$ only) differ by a factor of 5-10. Examples :

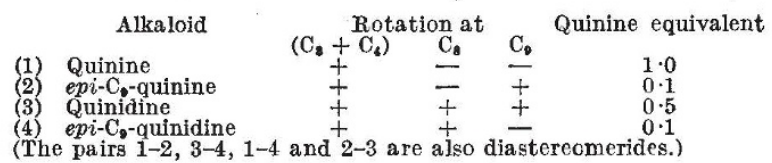

In view of these data, it is significant that the two isomers of Ainley and King's substance (II) have rather different antiplasmodial activity (the isomer of m.p. $162-163^{\circ}$ about the same as quinine, that of m.p. $187-188^{\circ}$ only 25 per cent of it). As, according to the synthesis, the two substances are diastereomides, one is tempted to suggest that the signs of rotation at the two asymmetric carbon atoms (which correspond to $\mathrm{C}_{8}$ and $\mathrm{C}_{8}$ in the quinine formula) are the same in the more active isomer of m.p. $162-163^{\circ}$ and are different in the less active isomer

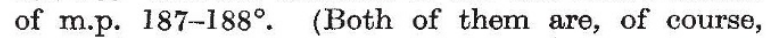
racemates.)

The somewhat unusual regularities which emerge from the accumulated experimental material seem to suggest that-in contradistinction with so many other biological effects-an optically active selective chemoceptor? is not responsible for antiplasmodial activity.
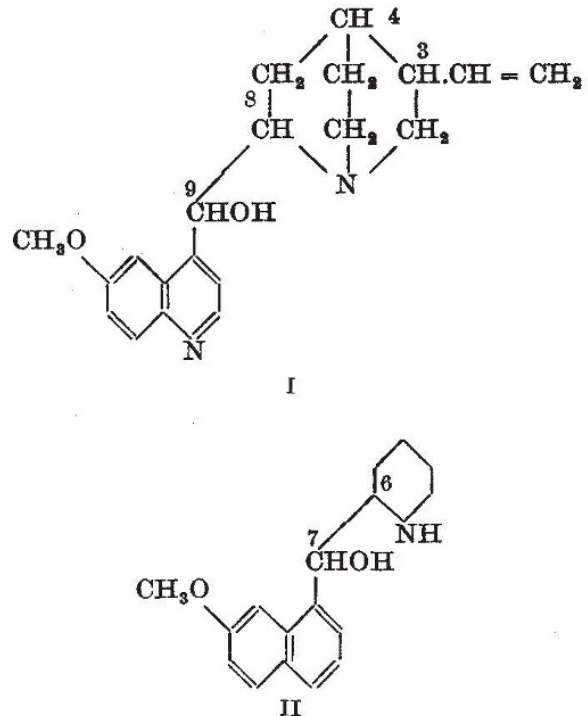

M. Negman.

Daniel Sieff Research Institute,

Rehovoth.

Sept. 1.

1 Goodman and Gilman, "The Pharmacological Basis of Therapeutics" 905 (New York, 1943)

' Buttle, Henry, Solomon, Trevan and Gibbs, Biochem. J., 32, 47 (1938).

"See Henry, Solomon and Gibbs, J. Chem. Soc., 592 (1937), in conjunction with King and Palmer, J. Chem. Soc., 121, 2577 (1922). - Ainley and King, Proc. Roy. Soc., B, 125, 60 (1938).

-Thron and Dirscherl, Liebigs Ann., 521, 48 (1935).

- Henry, Chem. and Ind., 55 T, 111 (1936).

"Cushny, "Biological Relations of Optically Isomeric Substances" (Baltimore, 1926)

\section{Antibiotic Action of Aspergillus fumigatus against Mycobacterium tuberculosis}

MANY papers have been published on the antagonism of certain micro-organisms against acid-fast bacteria, particularly Mycobacterium tuberculosis ${ }^{1,2,3,4}$, recording varying results. I have tested during the past year various fungi for antagonism to acid-fast bacteria and have found Aspergillus fumigatus No. 367 of the National Collection of Type Culture to be the most effective.

Of the different media tested, Czapek-Dox medium with 2 per cent tryptone, 4 per cent brown sugar and 5 per cent glycerine yielded the best results. The active filtrate will be referred to as 'aspergillin'. The antibacterial power of the culture, which reaches its maximum in about 15-20 days, was determined by making serial dilutions of aspergillin in fresh broth with 5 per cent glycerine contained in 50 c.c. conical flasks, or 2-oz. flat medicine bottles. Then these were inoculated with a small amount (1-2 mgm.) of a young culture of acid-fast bacteria on glycerol broth or egg medium. In each test control cultures were also seeded. The cultures were incubated at $37^{\circ} \mathrm{C}$.

The degree of growth in the various dilutions of aspergillin was compared with the growth on the control cultures at intervals of time. The final examinations of the cultures were made after 4-6 weeks in the case of Mycobacterium tuberculosis bovine and human type, with Mycobacterium tuberculosis avian type and Mycobacterium phlei after six days. For 